\title{
Is tranexamic acid going to CRASH the management of traumatic brain injury?
}

\author{
Fabio Silvio Taccone ${ }^{1 *}$ (D) Giuseppe Citerio $^{2}$ and Nino Stocchetti ${ }^{3}$
}

(c) 2019 Springer-Verlag GmbH Germany, part of Springer Nature

In the recent CRASH-3 randomized trial [1], early $(<3 \mathrm{~h})$ administration of tranexamic acid (TXA) was associated with a non-significant reduction [i.e., from 19.8 to $18.5 \%$; relative risk, RR 0.94 (95\% CI 0.86-1.02)] of head injuryrelated 28-day mortality in patients with isolated traumatic brain injury (TBI). However, there was a significant reduction in head injury-related mortality when TXA was administered within $3 \mathrm{~h}$ to patients with mild-tomoderate [from 7.5 to $5.8 \%$, RR 0.78 (95\% CI 0.64-0.95)] but not severe [from 40.1 to $39.6 \%$, RR 0.99 (95\% CI 0.911.07)] TBI. Major extra-cranial bleeding was an exclusion criterion, as the administration of TXA, which inhibits the enzymatic breakdown of fibrinolysis, has been proven to be effective to reduce mortality in this setting [2].

The trial has been an impressive endeavor, with almost 13,000 patients randomized in 175 hospitals from 29 countries. A large proportion of patients was included in low- or middle-income countries, where TBI represents an increasing burden and trials are rarely performed. The rate of follow-up was also incredibly high, with information on the primary outcome being available for $99.2 \%$ of patients. The authors concluded that "TXA treatment within $3 \mathrm{~h}$ of injury reduced TBI-related death" but correctly underlined in the discussion that the confidence intervals were wide, i.e., compatible both with a substantial reduction in TBI-related death or little or no benefit [1]. In contrast to this prudent comments, the accompanying editorial [3] as well as the infographic distributed by the Lancet within social media presented TXA as "a win for patients with TBI" and as a therapy that "could

*Correspondence: ftaccone@ulb.ac.be

${ }^{1}$ Department of Intensive Care, Hôpital Erasme, Route de Lennik, 808, 1070 Brussels, Belgium

Full author information is available at the end of the article save 1 in 5 people who would have died following a mild or moderate traumatic brain injury".

This enthusiasm is concerning because, at first glance, this was a "neutral" study, as the intervention was associated with a very limited (around 1\%) and non-significant reduction of the primary outcome (head injury-related mortality at 28 days). The number of TBI patients to be treated to obtain an additional survivor by TXA therapy was 66 in the study cohort excluding patients with Glasgow Coma Score (GCS) of three or bilateral unreactive pupils and 71 in mild-to-moderate injury, which suggests a relatively limited drug effect on patients' outcome. Several additional issues are worth discussing. First, a significant reduction in mortality was observed exclusively in subgroup analyses and, regrettably, no benefit could be demonstrated in severe cases. Moreover, no reason was provided for the change from "all-cause mortality" to "head injury-related mortality", which could be more challenging to define in a pragmatic trial, as primary outcome of the study. Also, assessment of mortality at 28 days from injury could be criticized, as some patients could die at a later stage because of withdrawal of lifesustaining therapies due to persistent poor clinical status and/or life-threatening complications. Second, the study protocol was modified over time, although blindly to interim data analyses, with a reduction in the time from injury to randomization from 8 to $3 \mathrm{~h}$; this excluded from the final analysis $28 \%$ of the recruited patients. Third, the small reduction in mortality by TXA was even less significant in low- and middle-income countries, where a simple and cheap intervention would be widely applicable. Fourth, as important as survival can be, quality of survival is extremely important, due to the growing evidence that TBI causes long-term consequences $[4,5]$. In the CRASH-3 study, disability assessment was performed using the Disability Rating Scale and an outcome measure designed by relatives: both methods showed

\section{Springer}




\section{TRAUMATIC BRAIN INJURY}

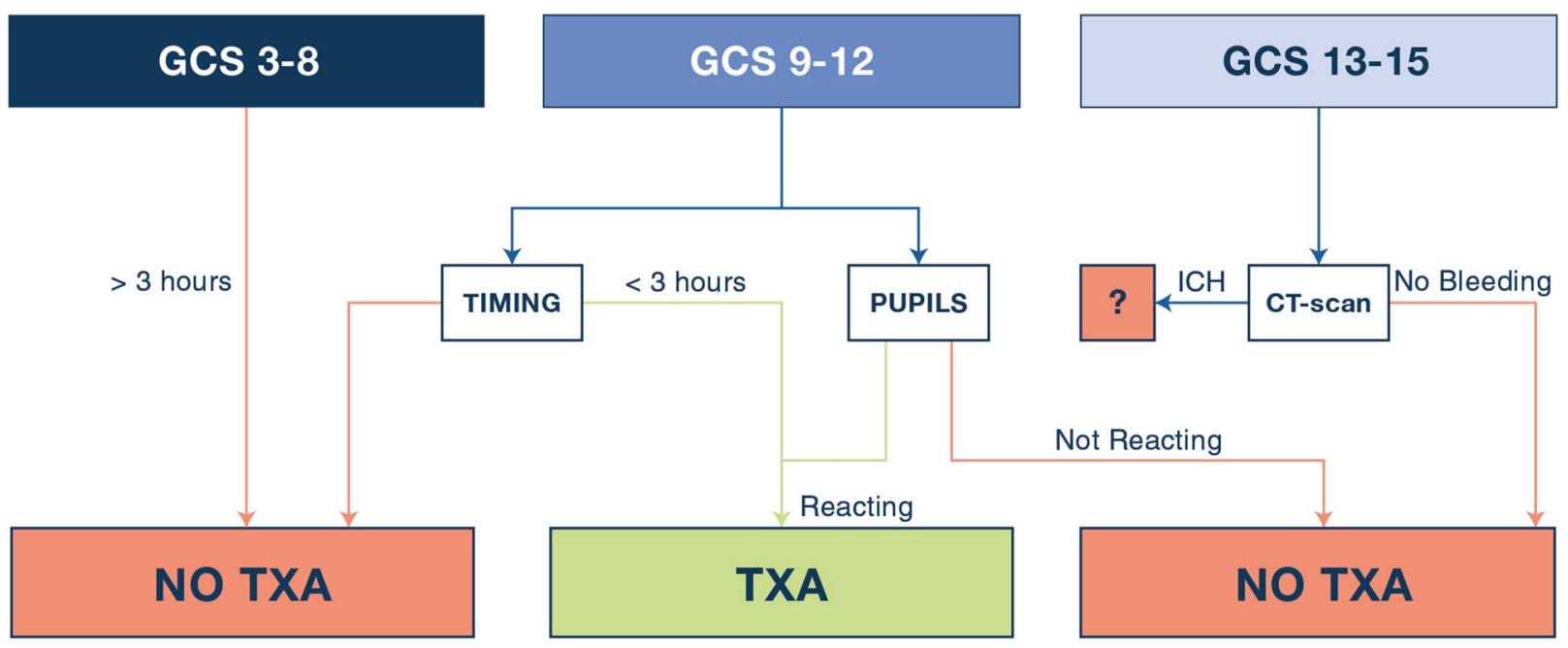

Fig. 1 Personal interpretation of the most effective use of tranexamic acid (TXA) in isolated traumatic brain injury patients. GCS Glasgow Coma Scale

no differences between groups. However, the timing of those assessments was not specifically reported. Fifth, as the occurrence of thrombotic events was similar in the TXA and placebo groups (1.5 vs. $1.3 \%$, respectively), the drug was considered "safe". However, this complication was recorded only if clinically obvious, at the risk of being largely underestimated. Other trials, with systematic deep venous thrombosis screening, have shown that almost $20 \%$ of severe TBI patients might present this complication, in the absence of pro-coagulant medications [6]. Sixth, patients with GCS of 13-15 (i.e., a very low risk of mortality) and a positive CT-scan (i.e., visible intra-cranial hemorrhage) were also included but no data are specifically reported for those. Moreover, the authors initially proposed a subgroup analysis for mild, moderate and severe TBI; the combination of mild and moderate TBI was not scheduled in the published outcome analyses plan [7]. Seventh, no mechanistic effects of TXA were proven, as data on the progression of the intra-cranial bleeding on CT-scans or on coagulation parameters, which are the essential markers and targets of this drug, were not available.

Notwithstanding the long list of open questions, the CRASH-3 trial provides results that deserve serious consideration. Patients who were randomized within $3 \mathrm{~h}$ and suffered less severe brain damage (i.e., GCS 9-15 or both pupils reactive) showed a tangible mortality benefit. Time and severity seem, therefore, the two key parameters underlying the findings: earlier administration could decrease the extent of the bleeding before the hemorrhagic volume may become dangerous. On the contrary, late administration can be useless, once a significant hemorrhage has accumulated, or even dangerous, as suggested in the previous CRASH-2 and WOMAN studies $[2,8]$. Accordingly, hemorrhage reduction in patients with limited brain damage (higher GCS and preserved pupillary reactivity) could further protect from secondary deterioration, while it could not benefit a brain with extended post-traumatic damage.

Based on this trial, should we include early TXA administration in our practice? The answer, in our opinion, is multifaceted and has been summarized as our personal interpretation of the trial in Fig. 1. TXA is not indicated for the severe cases, who are the majority of patients arriving in the ICU. TXA administration was not effective in comatose patients and/or those with pupillary abnormalities. Information is at the moment too scarce for patients with a mild TBI (i.e., GCS 13-15); however, evidence of initial bleeding at the CT-scan may justify TXA administration. Finally, we believe there is enough evidence, after the CRASH-3 study, to support early (i.e., within the first $3 \mathrm{~h}$ after injury) TXA infusion for 
moderate TBI patients, with a GCS 9-12 and preserved pupillary reactivity. This is, definitely, a change in clinical practice, but individualized in a subgroup of TBI patients in whom benefits may be expected.

What should not change for these patients, however, is the strategy that already saves lives of TBI patients everyday: preservation of physiological homeostasis by avoidance of secondary injury (i.e., hypoxemia, hypotension, hyponatremia, anemia, hyperthermia) coupled with meticulous clinical observation and CT-scan surveillance. In case of clinical deterioration a new CT-scan can be life-saving $[9,10]$, by disclosing the threat of expanding hematomas and making prompt surgical evacuation possible, whenever needed.

\section{Author details}

${ }^{1}$ Department of Intensive Care, Hôpital Erasme, Route de Lennik, 808, 1070 Brussels, Belgium. ${ }^{2}$ Neurointensive Care Unit, San Gerardo Hospital, ASST-Monza, Monza, MB, Italy. ${ }^{3}$ Department of Physiopathology and Transplant, Milan University-Neuro ICU, Fondazione IRCCS Cà Granda Ospedale Maggiore Policlinico, Milan, Italy.

\section{Compliance with ethical standards}

\section{Conflicts of interest}

None to declare.

\section{Publisher's Note}

Springer Nature remains neutral with regard to jurisdictional claims in published maps and institutional affiliations.

Received: 1 November 2019 Accepted: 21 November 2019 Published online: 9 December 2019

\section{References}

1. CRASH-3 trial collaborators (2019) Effects of tranexamic acid on death, disability, vascular occlusive events and other morbidities in patients with acute traumatic brain injury (CRASH-3): a randomised, placebo-controlled trial. Lancet. https://doi.org/10.1016/s0140-6736(19)32233-0

2. CRASH-2 trial collaborators, Shakur H, Roberts I, Bautista R et al (2010) Effects of tranexamic acid on death, vascular occlusive events, and blood transfusion in trauma patients with significant haemorrhage (CRASH-2): a randomised, placebo-controlled trial. Lancet 376(9734):23-32. https:// doi.org/10.1016/s0140-6736(10)60835-5

3. Cap AP (2019) CRASH-3: a win for patients with traumatic brain injury. Lancet. https://doi.org/10.1016/s0140-6736(19)32312-8

4. Perry DC, Sturm VE, Peterson MJ et al (2016) Association of traumatic brain injury with subsequent neurological and psychiatric disease: a meta-analysis. J Neurosurg 124(2):511-526. https://doi. org/10.3171/2015.2.JNS14503

5. Griesbach GS, Masel BE, Helvie RE, Ashley MJ (2018) The impact of traumatic brain injury on later life: effects on normal aging and neurodegenerative diseases. J Neurotrauma 35(1):17-24. https://doi.org/10.1089/ neu.2017.5103

6. Skrifvars MB, Bailey M, Presneill J et al (2017) Venous thromboembolic events in critically ill traumatic brain injury patients. Intensive Care Med 43(3):419-428. https://doi.org/10.1007/s00134-016-4655-2

7. Roberts I, Belli A, Brenner A et al (2018) Tranexamic acid for significant traumatic brain injury (The CRASH-3 trial): statistical analysis plan for an international, randomised, double-blind, placebo-controlled trial. Welcome Open Res 3:86

8. Woman Trial Collaborators (2017) Effect of early tranexamic acid administration on mortality, hysterectomy, and other morbidities in women with post-partum haemorrhage (WOMAN): an international, randomised, double-blind, placebo-controlled trial. Lancet 389(10084):2105-2116. https://doi.org/10.1016/S0140-6736(17)30638-4

9. Servadei F, Murray GD, Penny K et al (2000) The value of the "worst" computed tomographic scan in clinical studies of moderate and severe head injury. European brain injury consortium. Neurosurgery 46(1):70-77. https //doi.org/10.1097/00006123-200001000-00014

10. Hawryluk GWJ, Aguilera S, Buki A et al (2019) A management algorithm for patients with intracranial pressure monitoring: the Seattle International Severe Traumatic Brain Injury Consensus Conference (SIBICC). Intensive Care Med. https://doi.org/10.1007/s00134-019-05805-9 